\title{
Espessura do processo alveolar da região anterior da maxila e mandíbula em pacientes com discrepância óssea ântero-posterior
}

\author{
Rachel de Mattos Garcia*, Cristiane Aparecida de Assis Claro **, Rosana Villela Chagas ${ }^{\star \star \star}$,

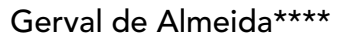

\begin{abstract}
Resumo
O presente estudo teve como objetivo avaliar a espessura do processo alveolar da região anterior da maxila e mandíbula em pacientes portadores de discrepâncias ântero-posteriores. Após a seleção de telerradiografias laterais de cinqüenta e dois pacientes entre idades de sete e 13 anos, mediu-se a espessura do processo alveolar da região anterior da maxila e mandíbula. Todos os pacientes apresentavam o ângulo do plano mandibular entre vinte e trinta graus e discrepância óssea ântero-posterior entre maxila e mandíbula. Após a análise estatística (teste qui-quadrado) observou-se que não houve dependência entre a espessura do processo alveolar da maxila e mandíbula e a idade do paciente. Todavia houve dependência entre o tipo de má oclusão e a espessura de osso vestibular na região anterior da maxila. Os pacientes portadores de má oclusão de Classe III apresentaram maior porcentagem de redução de osso vestibular da região anterior da maxila quando comparados aos pacientes Classe II. Os pacientes com tendência ao crescimento vertical apresentaram a dimensão reduzida de osso lingual da maxila e osso vestibular da mandíbula.
\end{abstract}

Palavras-chave: Espessura do processo alveolar. Má oclusão. Tipo facial. Perda iatrogênica.

\section{INTRODUÇÃO}

O delineamento dos limites do tratamento ortodôntico é fator importante a ser considerado, principalmente em casos ortodônticos-cirúrgicos limítrofes. Este delineamento realizado antes do tratamento seria extremamente benéfico. O planejamento do tratamento ortodôntico poderia ser bem valorizado, especialmente em situações nas quais a discrepância esquelética é severa ou onde um ou ambos os arcos podem acomodar somente reposicionamento limitado do dente. As tábuas corticais vestibulares e linguais ao nível do ápice dos incisivos podem representar os limites anatômicos da movimentação dentária 5 .

* Especialista em Odontopediatria pelo Departamento de Odontologia da Universidade de Taubaté, Especialista em Ortodontia e

Ortopedia Facial pelo Departamento de Odontologia da Universidade de Taubaté.

** Especialista em Ortodontia, Mestre em Odontologia, Professora Colaboradora Assistente da Disciplina de Ortodontia do Departamento de Odontologia da Universidade de Taubaté, Professora do Curso de Especialização em Ortodontia e Ortopedia Facial do Departamento de Odontologia da Universidade de Taubaté, Doutoranda em Ortodontia na Universidade de São Paulo.

*** Especialista em Ortodontia, Mestre e Doutora em Odontologia, Professora responsável da Disciplina de Ortodontia do Departamento de Odontologia da Universidade de Taubaté, Professora do Curso de Especialização em Ortodontia e Ortopedia Facial do Departamento de Odontologia da Universidade de Taubaté.

*** Mestre e Doutor em Odontologia e Coordenador do Curso de Especialização em Ortodontia e Ortopedia Facial do Departamento de Odontologia da Universidade de Taubaté. 
A movimentação dentária pode ser limitada em pacientes com osso alveolar de pequena espessura e estes pacientes provavelmente experimentam maior incidência de seqüela iatrogênica. Os dentes anteriores apresentam maior porcentagem de fenestrações e deiscência quando comparados aos dentes posteriores ${ }^{6,11,16,22}$. O contorno ósseo normalmente acompanha a proeminência das raízes, com depressões verticais que se estreitam para a margem. A anatomia do osso alveolar varia entre os pacientes, com importantes implicações clínicas. A altura e a espessura das corticais vestibulares e linguais são afetadas pelo alinhamento dos dentes, pela angulação das raízes nos ossos e pelas forças

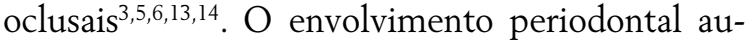
menta significantemente as tensões produzidas no ligamento periodontal, especialmente quando forças de inclinação são aplicadas ${ }^{4}$. A porção cervical do osso alveolar é, às vezes, consideravelmente mais espessa na face vestibular, aparentemente como reforço contra as forças oclusais.

Os limites anatômicos, impostos pelas tábuas corticais do processo alveolar ao nível dos ápices dos incisivos, podem ser considerados como "paredes ortodônticas", bem como zona de perigo para as seqüelas desfavoráveis ${ }^{3,5}$.

A questão é saber quando o ortodontista deve considerar se uma movimentação ortodôntica dentária é suficiente para corrigir a má oclusão, e se esta movimentação pode ser realizada com perda tecidual mínima por iatrogenia. Em tratamentos ortodônticos sempre se busca estética facial aceitável com mínima perda tecidual. Este comprometimento estético talvez seja a motivação mais importante que leva o paciente a buscar o tratamento ortodôntico, principalmente nas sociedades ocidentais, onde a aparência dentofacial tem papel fundamental nas interrelações pessoais e que a população em geral apresenta tendências positivas para indivíduos com faces bem proporcionais.

Radiografias cefalométricas foram introduzidas por Broadbent em 1931 e tem sido importante recurso utilizado pelos ortodontistas para determi- nar os limites da movimentação dentária, e se a quantidade de osso alveolar disponível é suficiente para a movimentação segura dos incisivos, a fim de corrigir as discrepâncias ântero-posteriores. É importante lembrar que as radiografias cefalométricas apresentam visão bidimensional, porém os limites reais podem ser mais estreitos do que na imagem obtida. Para um melhor diagnóstico são consideradas outras técnicas radiográficas, como por exemplo radiografias periapicais, tomografias computadorizadas e não é dispensável a análise cuidadosa dos modelos de estudo $6,8,9,12,17,20$.

As inclinações compensatórias dos incisivos superiores e inferiores resultam em relação normal dos incisivos, apesar de algumas variações na posição sagital da mandíbula ${ }^{8,9}$. A cirurgia ortognática é geralmente solicitada para melhorar as relações esqueléticas e harmonia do tecido mole, bem como estabelecer oclusão estável. Entretanto há casos em que, por algum motivo, o paciente recusa a cirurgia ortognática e o profissional busca a correção ortodôntica por meio de compensações dentárias. Nos pacientes que apresentam má oclusão de Classe II esquelética, a compensação é realizada às custas da vestibularização dos dentes ântero-inferiores e lingualização dos dentes ântero-superiores. Nos pacientes com má oclusão de Classe III, a compensação dentária é realizada com a lingualização dos dentes ânteroinferiores e vestibularização dos dentes ânterosuperiores. Nos casos de pacientes tratados com compensação dentária, o ortodontista pretende obter equilíbrio funcional, estético, e oclusão próxima do ideal. Os resultados dos tratamentos que envolvem compensações, geralmente são frustantes, pois estão mais sujeitos a recidivas e iatrogenias.

\section{MATERIAL E MÉTODO}

Obtida aprovação do Comitê de Ética, este estudo retrospectivo analisou as telerradiografias laterais de 56 pacientes, entre sete e 13 anos de idade, sendo 27 do gênero feminino e 29 do mas- 
culino. Do total de pacientes avaliados, 36 pacientes apresentavam má oclusão de Classe II e vinte má oclusão de Classe III. A amostra foi selecionada de arquivos da clínica de pós-graduação da Universidade de Taubaté e dos consultórios particulares dos professores do Curso de Especialização em Ortodontia da mesma Universidade.

O parâmetro para idade entre sete e 13 anos foi definido pelo fato desses pacientes apresentarem-se em fase de crescimento craniofacial. Esta escala de idade permitiu a divisão em três grupos (7 a 9 anos, 9 a 11 anos e 11 a 13 anos).

Os pacientes que apresentaram um ângulo ANB maior que $5^{\circ}$ (sugestivo de Classe II) e menor que $1^{\circ}$ (sugestivo de Classe III) foram incluídos nesta amostra. A medida do ângulo do plano mandibular (FMA) foi um dos critérios de exclusão da amostra, participando apenas os pacientes que apresentaram este ângulo com medidas entre $20^{\circ}$ e $30^{\circ}$. Quatro pacientes foram excluídos por apresentarem rizogênese incompleta (formação incompleta da raiz dentária) dos incisivos centrais permanentes, restringindo a amostra a 52 pacientes, sendo 25 do gênero feminino e 27 do masculino.

Das telerradiografias finalizadas, 33 pacientes apresentavam má oclusão de Classe II e 19 de Classe III esquelética.

Com a intenção de minimizar as possíveis alterações, somente foram incluídas as telerradiografias laterais obtidas no mesmo Instituto de Radiologia, que utilizou o cefalostato Orthophos cd Siemens. A distância do foco ao filme foi de $1,5 \mathrm{~m}$. Os pontos cefalométricos foram acessados por traçado manual.

Os cefalogramas foram traçados em folha de acetato, com grafite número $0,3 \mathrm{~mm}$, e para mensuração foram utilizados régua, esquadros e transferidor, todos da marca Desetec.

O desenho anatômico compreendeu as seguintes estruturas: sela túrcica, perfil da glabela e ossos nasais, bordas inferiores da órbita, meato acústico externo, maxila, mandíbula, incisivos superior e inferior, primeiros molares superior e inferior e perfil tegumentar. Os pontos cefalométricos utilizados foram transcritos por Interlandi ${ }^{7}(\mathrm{~N}, \mathrm{~S}, \mathrm{Po}$, Or, Go, M, ENP, p', Gn). Para medir a espessura do osso alveolar da maxila, traçou-se uma reta paralela ao plano palatino (ENA-p') passando pelo ápice do incisivo central superior (U) e denominados UA e UP, os segmentos dessa reta, relativos à região anterior e posterior, respectivamente. Para medir a espessura do osso alveolar da mandíbula, traçou-se uma reta paralela ao plano mandibular (Go-M) passando pelo ápice do incisivo central inferior (I) e denominados IA e IP, os segmentos dessa reta, relativos à região anterior e posterior, respectivamente (Fig. 1).

\section{Erro do método}

Parte-se do princípio que as medidas realizadas estão sujeitas a uma série de fatores que podem resultar em medidas não precisas. Assim, caso uma medida seja realizada diversas vezes, espera-se que haja uma pequena discrepância entre os valores medidos. No entanto, para que as medidas sejam consideradas consistentes, estas variações "não

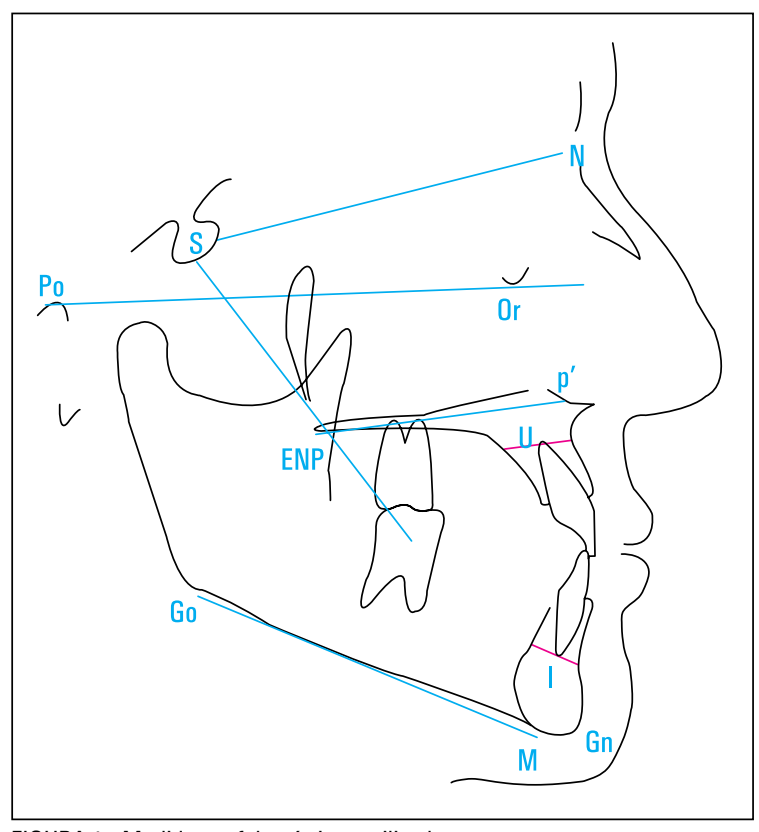

FIGURA 1 - Medidas cefalométricas utilizadas. 
controladas" não devem ser muito grandes.

Os erros que podem ser cometidos por ocasião da obtenção das medidas são: o erro sistemático e o erro aleatório (ou casual).

Para verificar se as mensurações das variáveis (SA, SP, IA, IP) estavam corretas, foram selecionados, de maneira aleatória, quinze pacientes, representando $28,84 \%$ da amostra, para que os traçados, bem como as medidas das grandezas cefalométricas, fossem repetidas.

Os dados referentes a cada repetição de cada uma das variáveis em estudo foram submetidos à análise de regressão linear simples. O modelo de regressão utilizado foi:

$Y_{i}=\beta_{0}+\beta_{1} X_{i}+E_{i}$

$Y_{i}=1^{a}$ medida

$\mathrm{X}_{\mathrm{i}}=2^{\mathrm{a}}$ medida

$\mathrm{E}_{\mathrm{i}}=$ erro

$\mathrm{i}=$ número do paciente

Se $\beta_{0}$ for igual a 0 (indicando que a reta passa pela origem) e $\beta_{1}$ for igual a 1 (indicando uma reta de $45^{\circ}$ ), pode-se constatar que não houve erro sistemático.

Para a avaliação deste tipo de erro, utiliza-se o coeficiente de correlação (r) ou o coeficiente de determinação $\left(\mathrm{r}^{2}\right)$. O coeficiente de determinação varia entre 0 e 1 . $O$ valor 0 indica que não há relação entre as duas medidas. Desta forma, caso o erro aleatório seja pequeno, espera-se que $r^{2}$ seja próximo a 1 .

\section{Método estatístico}

Os objetivos do trabalho foram buscar respos- tas para as seguintes questões:

1) Verificar a dependência estatística entre as medidas SA, SP, IA, IP e as Classes II e III.

2) Verificar a dependência estatística entre as medidas SA, SP, IA, IP com as medidas FMA e NSGn.

3) Verificar a dependência estatística entre a espessura do processo alveolar da região anterior da maxila e mandíbula com as idades dos pacientes.

O método estatístico realizou-se por meio do programa Minitab segundo Meyer e Krueger ${ }^{15}$, e o método adotado foi o teste de independência (teste do Qui-quadrado) realizado com as seguintes hipóteses:

$\mathrm{H}_{0}=$ as variáveis linha e coluna são independentes.

$\mathrm{H}_{1}=$ as variáveis linha e coluna são dependentes.

O nível de significância adotado neste trabalho foi de $5 \%$.

\section{RESULTADOS}

Os resultados do erro do método, verificado por meio de um modelo de regressão linear, considerando nível de significância de $5 \%$, pode ser observado na tabela 1 .

Considerando o nível de significância de 5\%, os resultados da análise de regressão linear não foram estatisticamente significativos. Desta forma, as retas estimadas passam pela origem e apresentam inclinação de $45^{\circ}$. Portanto, as medidas das grandezas cefalométricas obtidas no primeiro

Tabela 1 - Resultados da análise de regressão, para as medidas SA, SP, IA, IP.

\begin{tabular}{ccccccccc}
\hline & $\mathbf{r}^{2}$ & $\beta_{0}$ & $\mathbf{t}\left(\beta_{0}=\mathbf{0}\right)$ & $\boldsymbol{p}$-value & $\beta_{1}$ & $\mathbf{t}\left(\beta_{1}=\mathbf{1}\right)$ & $\boldsymbol{p}$-value \\
\hline SA & 0,92 & 0,32 & 0,31 & 0,38 & 0,92 & 0,47 & 0,38 & Ns \\
SP & 0,97 & $-1,03$ & 0,72 & 0,24 & 1,06 & 0,55 & 0,30 & Ns \\
IA & 0,98 & 0,44 & 0,84 & 0,21 & 0,95 & 0,67 & 0,26 & Ns \\
IP & 0,96 & 0,28 & 0,52 & 0,31 & 0,95 & 0,48 & 0,32 & Ns \\
\hline
\end{tabular}

Ns = não significativa, ${ }^{*} p<0,05,{ }^{* *} p<0,01$. 
traçado são estatisticamente iguais às observadas no segundo traçado, evidenciando que os erros sistemático e casual foram desprezíveis.

Para facilitar a interpretação dos resultados do método estatístico, as tabelas seguintes serão apresentadas, agrupando as medidas das espessuras dos processos alveolares (SA, SP, IA, IP) com as idades dos pacientes, as más oclusões esqueléticas Classes
II e III e as medidas FMA e NSGn.

As tabelas 2, 3, 4, e 5 apresentam as freqüências observadas referentes à espessura do processo alveolar da região anterior da maxila e mandíbula, considerando as idades dos pacientes, e a tabela 6 identifica a ausência de relação de dependência entre as espessuras dos processos alveolares e as idades dos pacientes.

Tabela 2 - Freqüência observada das medidas SA (subdivididas em três categorias), considerando as idades dos pacientes (subdivididas em três categorias).

\begin{tabular}{ccccc}
\hline SA & $\mathbf{2}$ e $\leq \mathbf{9}$ anos & $>\mathbf{9}$ e $\leq \mathbf{1 1}$ anos & $>\mathbf{1 1} \mathrm{e} \leq \mathbf{1 3}$ anos & TOTAL \\
\hline $\mathbf{2 , 5} / \mathbf{4 , 5} \mathbf{~ m m}$ & $3(5,8 \%)$ & $2(3,8 \%)$ & $10(19,2 \%)$ & $15(28,8 \%)$ \\
$\mathbf{5 , 0} / \mathbf{7 , 0} \mathbf{~ m m}$ & $5(9,6 \%)$ & $13(25 \%)$ & $9(17,3 \%)$ & $27(52 \%)$ \\
$\mathbf{7 , 5} / \mathbf{9 , 5} \mathbf{~ m m}$ & $1(1,9 \%)$ & $5(5,8 \%)$ & $4(7,8 \%)$ & $10(19,2)$ \\
TOTAL & $9(17,3 \%)$ & $20(38,5 \%)$ & $23(44,2 \%)$ & $52(100 \%)$ \\
\hline
\end{tabular}

Tabela 3 - Freqüência observada das medidas SP (subdivididas em três categorias), considerando as idades dos pacientes (subdivididas em três categorias).

\begin{tabular}{ccccc}
\hline $\mathbf{S P}$ & $\mathbf{2}$ e $\leq \mathbf{9}$ anos & $>\mathbf{9}$ e $\leq \mathbf{1 1}$ anos & $>\mathbf{1 1}$ e $\leq \mathbf{1 3}$ anos & TOTAL \\
\hline $\mathbf{5 , 0} / \mathbf{9 , 5} \mathbf{~ m m}$ & $2(3,8 \%)$ & $6(11,6 \%)$ & $7(13,5 \%)$ & $15(28,8 \%)$ \\
$\mathbf{1 0 , 0} / \mathbf{1 4 , 5} \mathbf{~ m m}$ & $5(9,6 \%)$ & $13(25 \%)$ & $13(25 \%)$ & $31(59,6 \%)$ \\
$\mathbf{1 5 , 0} / \mathbf{1 9}, \mathbf{5 m m}$ & $2(3,8 \%)$ & $1(1,9 \%)$ & $3(5,8 \%)$ & $6(11,6 \%)$ \\
TOTAL & $9(17,3 \%)$ & $20(38,5 \%)$ & $23(44,2 \%)$ & $52(100 \%)$ \\
\hline
\end{tabular}

Tabela 4 - Freqüência observada das medidas IA (subdivididas em três categorias), considerando as idades dos pacientes (subdivididas em três categorias).

\begin{tabular}{ccccc}
\hline IA & $\geq \mathbf{7}$ e $\leq \mathbf{9}$ anos & $>\mathbf{9}$ e $\leq \mathbf{1 1}$ anos & $>\mathbf{1 1}$ e $\leq \mathbf{1 3}$ anos & TOTAL \\
\hline $\mathbf{3 , 0} / \mathbf{5 , 0} \mathbf{~ m m}$ & $1(1,9 \%)$ & $6(11,6 \%)$ & $6(11,6 \%)$ & $13(25 \%)$ \\
$\mathbf{5 , 5} / \mathbf{7 , 5} \mathbf{~ m m}$ & $4(7,7 \%)$ & $10(19,2 \%)$ & $14(26,8 \%)$ & $28(53,8 \%)$ \\
$\mathbf{8 , 0} / \mathbf{1 0 , 0} \mathbf{~ m m}$ & $4(7,7 \%)$ & $4(7,7 \%)$ & $3(5,8 \%)$ & $11(21,2 \%)$ \\
TOTAL & $9(17,3 \%)$ & $20(38,5 \%)$ & $23(44,2 \%)$ & $52(100 \%)$ \\
\hline
\end{tabular}

Tabela 5 - Freqüência observada das medidas IP (subdivididas em três categorias), considerando as idades dos pacientes (subdivididas em três categorias).

\begin{tabular}{ccccc}
\hline $\mathbf{I P}$ & $\mathbf{2}$ e $\leq \mathbf{9}$ anos & $>\mathbf{9}$ e $\leq \mathbf{1 1}$ anos & $>\mathbf{1 1}$ e $\leq \mathbf{1 3}$ anos & TOTAL \\
\hline $\mathbf{2 , 0 / 3 , 5 ~} \mathbf{~ m m}$ & $1(1,9 \%)$ & $4(7,7 \%)$ & $3(5,8 \%)$ & $8(15,4 \%)$ \\
$\mathbf{4 , 0} / \mathbf{5 , 5} \mathbf{~ m m}$ & $6(11,6 \%)$ & $13(25 \%)$ & $17(32,6 \%)$ & $36(69,2 \%)$ \\
$\mathbf{6 , 0 / 7 , 5 ~} \mathbf{~ m m}$ & $2(3,8 \%)$ & $3(5,8 \%)$ & $3(5,8 \%)$ & $8(15,4 \%)$ \\
TOTAL & $9(17,3 \%)$ & $20(38,5 \%)$ & $23(44,2 \%)$ & $52(100 \%)$ \\
\hline
\end{tabular}


As tabelas 7, 8, 9 e 10 apresentam as freqüências observadas referentes à espessura do processo alveolar da região anterior da maxila e mandíbula, considerando as más oclusões esqueléticas de Classe II e III, e a tabela 11 identifica que apenas a espessura óssea da região anterior da maxila apresentou relação de dependência com as más oclu-

Tabela 6 - Teste de independência (qui-quadrado) entre as medidas SA, SP, IA, IP e as idades dos pacientes.

\begin{tabular}{ccc}
\hline Teste & $p$-value & Dep (D) / Indep (I) \\
\hline SA x idade & 0,176 & $\mathrm{I}$ \\
SP x idade & 0,735 & $\mathrm{I}$ \\
IA x idade & 0,360 & $\mathrm{I}$ \\
IP x idade & 0,916 & $\mathrm{I}$ \\
\hline
\end{tabular}

sões de Classe II e III esqueléticas. A freqüência de pacientes com má oclusão de Classe III e redução de osso vestibular ao ápice do incisivo central superior é maior quando comparada à freqüência de pacientes com má oclusão de Classe II.

As tabelas 12, 13, 14 e 15 apresentam as freqüências observadas referentes à espessura do processo alveolar da região anterior da maxila e mandíbula com a medida NSGn, variando entre 60 e $68,2^{\circ}$, e a tabela 16 identifica a relação de dependência entre as espessuras ósseas da região póstero-superior (SP) e ântero-inferior (IA) com a medida NSGn. A freqüência de pacientes com tendência ao crescimento vertical e redução de osso na lingual ao ápice do incisivo central superior é maior quando comparada à freqüência de

Tabela 7 - Freqüência observada das medidas SA (subdivididas em três categorias), considerando as más oclusões esqueléticas de Classe II e III.

\begin{tabular}{ccccc}
\hline \multicolumn{5}{c}{ SA } \\
\hline Classes & $\mathbf{2 , 5 / 4 , 5 ~} \mathbf{~ m m}$ & $\mathbf{5 , 0 / 7 , 0 ~} \mathbf{~ m m}$ & $\mathbf{7 , 5} / \mathbf{9 , 5} \mathbf{~ m m}$ & TOTAL \\
\hline II & $8(24,2 \%)$ & $22(66,7 \%)$ & $3(9,1 \%)$ & $33(63,5 \%)$ \\
III & $7(36,8 \%)$ & $6(31,6 \%)$ & $6(31,6 \%)$ & $19(36,5 \%)$ \\
TOTAL & $15(28,8 \%)$ & $28(53,8 \%)$ & $9(17,4 \%)$ & $52(100 \%)$ \\
\hline
\end{tabular}

Tabela 8 - Freqüência observada das medidas SP (subdivididas em três categorias), considerando as más oclusões esqueléticas de Classe II e III.

\begin{tabular}{|c|c|c|c|c|}
\hline \multicolumn{5}{|c|}{ SP } \\
\hline Classes & $5,0 / 9,5 \mathrm{~mm}$ & $10,0 / 14,5 \mathrm{~mm}$ & 15,0 / 19,5 mm & TOTAL \\
\hline II & $9(27,3 \%)$ & $20(60,6 \%)$ & $4(12,1 \%)$ & $33(63,5 \%)$ \\
\hline III & $6(31,6 \%)$ & $12(63,1 \%)$ & $1(5,3 \%)$ & $19(36,5 \%)$ \\
\hline TOTAL & $15(28,8 \%)$ & $32(61,5 \%)$ & $5(9,6 \%)$ & $52(100 \%)$ \\
\hline
\end{tabular}

Tabela 9 - Freqüência observada das medidas IA (subdivididas em três categorias), considerando as más oclusões esqueléticas de Classe II e III.

\begin{tabular}{ccccc}
\hline \multicolumn{5}{c}{ IA } \\
\hline Classes & $\mathbf{3 , 0} / \mathbf{5 , 0} \mathbf{~ m m}$ & $\mathbf{5 , 5} / \mathbf{7 , 5} \mathbf{~ m m}$ & $\mathbf{8 , 0} / \mathbf{1 0 , 0} \mathbf{~ m m}$ & TOTAL \\
\hline II & $7(21,2 \%)$ & $21(63,6 \%)$ & $5(15,2 \%)$ & $33(63,5 \%)$ \\
III & $6(31,6 \%)$ & $7(36,8 \%)$ & $6(31,6 \%)$ & $19(36,5 \%)$ \\
TOTAL & $15(28,8 \%)$ & $32(61,5 \%)$ & $5(9,6 \%)$ & $52(100 \%)$ \\
\hline
\end{tabular}


Tabela 10 - Freqüência observada das medidas IP (subdivididas em três categorias), considerando as más oclusões esqueléticas de Classe II e III.

\begin{tabular}{|c|c|c|c|c|}
\hline \multicolumn{5}{|c|}{ IP } \\
\hline Classes & $2,0 / 3,5 \mathrm{~mm}$ & $4,0 / 5,5 \mathrm{~mm}$ & $6,0 / 7,5 \mathrm{~mm}$ & TOTAL \\
\hline II & $7(21,2 \%)$ & $20(60,6 \%)$ & $6(18,2 \%)$ & $33(63,5 \%)$ \\
\hline III & $1(5,3 \%)$ & $16(84,2 \%)$ & $2(10,5 \%)$ & $19(36,5 \%)$ \\
\hline TOTAL & $8(15,4 \%)$ & $36(69,2 \%)$ & $8(15,4 \%)$ & $52(100 \%)$ \\
\hline
\end{tabular}

Tabela 11 - Teste de independência (qui-quadrado) entre as medidas SA, SP, IA e IP e as Classes esqueléticas II e III.

\begin{tabular}{ccc}
\hline Teste & $\boldsymbol{p}$-value & Dep (D) / Indep (I) \\
\hline SA x Classe & 0,031 & $\mathrm{D}$ \\
SP x Classe & 0,712 & $\mathrm{I}$ \\
IA x Classe & 0,160 & $\mathrm{I}$ \\
IP x Classe & 0,181 & $\mathrm{I}$ \\
\hline
\end{tabular}

Tabela 12 - Freqüência observada das medidas SA (subdivididas em três categorias), considerando a medida NSGn (subdivididas em duas categorias).

\begin{tabular}{|c|c|c|c|}
\hline \multirow{2}{*}{ SA } & \multicolumn{2}{|c|}{ NSGn } & \multirow{2}{*}{ TOTAL } \\
\hline & $60.0^{\circ} / 68.2^{\circ}$ & $68.3^{\circ} / 76.5^{\circ}$ & \\
\hline $2,5 / 4,5 \mathrm{~mm}$ & $10(19,2 \%)$ & $5(9,7 \%)$ & $15(28,8 \%)$ \\
\hline $5,0 / 7,0 \mathrm{~mm}$ & $13(25,0 \%)$ & $15(28,8 \%)$ & $28(53,8 \%)$ \\
\hline 7,5 / 9,5 mm & $7(13,5 \%)$ & $2(3,8 \%)$ & $9(17,4 \%)$ \\
\hline TOTAL & $30(57,7 \%)$ & $22(42,3 \%)$ & $52(100 \%)$ \\
\hline
\end{tabular}

Tabela 14 - Freqüência observada das medidas IA (subdivididas em três categorias), considerando a medida NSGn (subdivididas em duas categorias).

\begin{tabular}{cccc}
\hline \multicolumn{4}{c}{ NSGn } \\
\hline IA & $\mathbf{6 0 . 0 ^ { \circ } / \mathbf { 6 8 . 2 }}$ & $\mathbf{6 8 . 3 ^ { \circ }} / \mathbf{7 6 . 5 ^ { \circ }}$ & TOTAL \\
\hline $\mathbf{3 , 0} / \mathbf{5 , 0} \mathbf{~ m m}$ & $5(9,7 \%)$ & $8(15,4 \%)$ & $13(25,0 \%)$ \\
$\mathbf{5 , 5} / \mathbf{7 , 5} \mathbf{~ m m}$ & $15(28,8 \%)$ & $13(25,0 \%)$ & $28(53,8 \%)$ \\
$\mathbf{8 , 0} / \mathbf{1 0 , 0} \mathbf{~ m m}$ & $10(19,2 \%)$ & $1(1,9 \%)$ & $11(21,2 \%)$ \\
TOTAL & $30(57,7 \%)$ & $22(42,3 \%)$ & $52(100 \%)$ \\
\hline
\end{tabular}

Tabela 13 - Freqüência observada das medidas SP (subdivididas em três categorias), considerando a medida NSGn (subdivididas em duas categorias).

\begin{tabular}{|c|c|c|c|}
\hline \multirow{2}{*}{ SP } & \multicolumn{2}{|c|}{ NSGn } & \multirow{2}{*}{ TOTAL } \\
\hline & $60.0^{\circ} / 68.2^{\circ}$ & $68.3^{\circ} / 76.5^{\circ}$ & \\
\hline $5,0 / 9,5 \mathrm{~mm}$ & $3(5,8 \%)$ & $12(23,0 \%)$ & $15(28,8 \%)$ \\
\hline $10,0 / 14,5 \mathrm{~mm}$ & $22(42,3 \%)$ & $10(19,2 \%)$ & $28(53,8 \%)$ \\
\hline $15,0 / 19,5 \mathrm{~mm}$ & $5(9,7 \%)$ & $0(0 \%)$ & $9(17,4 \%)$ \\
\hline TOTAL & $30(57,7 \%)$ & $22(42,3 \%)$ & $52(100 \%)$ \\
\hline
\end{tabular}

Tabela 15 - Freqüência observada das medidas IP (subdivididas em três categorias), considerando a medida NSGn (subdivididas em duas categorias).

\begin{tabular}{cccc}
\hline \multicolumn{4}{c}{ NSGn } \\
\hline IP & $\mathbf{6 0 . 0}^{\circ} / \mathbf{6 8 . 2 ^ { \circ }}$ & $\mathbf{6 8 . 3 ^ { \circ }} / \mathbf{7 6 . 5 ^ { \circ }}$ & TOTAL \\
\hline $\mathbf{2 , 0} / \mathbf{3 , 5} \mathbf{~ m m}$ & $4(7,7 \%)$ & $4(7,7 \%)$ & $8(15,4 \%)$ \\
$\mathbf{4 , 0} / \mathbf{5 , 5} \mathbf{~ m m}$ & $20(38,4 \%)$ & $16(30,8 \%)$ & $36(69,2 \%)$ \\
$\mathbf{6 , 0} / \mathbf{7 , 5} \mathbf{~ m m}$ & $5(9,9 \%)$ & $3(5,8 \%)$ & $8(15,4 \%)$ \\
TOTAL & $29(55,8 \%)$ & $23(44,2 \%)$ & $52(100 \%)$ \\
\hline
\end{tabular}


Tabela 16 - Teste de independência (qui-quadrado) entre as medidas SA, SP, IA, IP e a medida NSGn.

\begin{tabular}{ccc}
\hline Teste & $p$-value & Dep (D) / Indep (I) \\
\hline SA x NSGn & 0,179 & I \\
SP x NSGn & 0,001 & $\mathrm{D}$ \\
IA x NSGn & 0,028 & $\mathrm{D}$ \\
IP x NSGn & 0,880 & $\mathrm{I}$ \\
\hline
\end{tabular}

Tabela 18 - Freqüência observada das medidas SP (subdivididas em três categorias), considerando a medida FMA (subdividida em duas categorias).

\begin{tabular}{|c|c|c|c|}
\hline \multirow{2}{*}{ SP } & \multicolumn{2}{|c|}{ FMA } & \multirow{2}{*}{ TOTAL } \\
\hline & $20.0^{\circ} / 25.0^{\circ}$ & $25.1^{\circ} / 30.1^{\circ}$ & \\
\hline $5,0 / 9,5 \mathrm{~mm}$ & $1(1,9 \%)$ & $14(26,9 \%)$ & $15(28,8 \%)$ \\
\hline $10,0 / 14,5 \mathrm{~mm}$ & $17(32,8 \%)$ & $15(28,8 \%)$ & $32(61,6 \%)$ \\
\hline $15,0 / 19,5 \mathrm{~mm}$ & $4(7,7 \%)$ & $1(1,9 \%)$ & $5(9,6 \%)$ \\
\hline TOTAL & $22(42,3 \%)$ & $30(57,7 \%)$ & $52(100 \%)$ \\
\hline
\end{tabular}

Tabela 20 - Freqüência observada das medidas IP (subdivididas em três categorias), considerando a medida FMA (subdividida em duas categorias).

\begin{tabular}{|c|c|c|c|}
\hline \multirow{2}{*}{ IP } & \multicolumn{2}{|c|}{ FMA } & \multirow{2}{*}{ TOTAL } \\
\hline & $20.0^{\circ} / 25.0^{\circ}$ & $25.1^{\circ} / 30.1^{\circ}$ & \\
\hline $2,0 / 3,5 \mathrm{~mm}$ & $4(7,7 \%)$ & $4(7,7 \%)$ & $8(15,4 \%)$ \\
\hline $4,0 / 5,5 \mathrm{~mm}$ & $15(28,8 \%)$ & $21(40,4 \%)$ & $36(69,2 \%)$ \\
\hline $6,0 / 7,5 \mathrm{~mm}$ & $3(5,8 \%)$ & $5(9,6 \%)$ & $8(15,4 \%)$ \\
\hline TOTAL & $22(42,3 \%)$ & $30(57,7 \%)$ & $52(100 \%)$ \\
\hline
\end{tabular}

pacientes com tendência ao crescimento horizontal. A freqüência de pacientes com tendência ao crescimento vertical e redução de osso na vestibular ao ápice do incisivo central inferior é maior quando comparada à frequiência de pacientes com tendência ao crescimento horizontal.

As tabelas 17, 18, 19 e 20 apresentam as freqüências observadas referentes à espessura do processo alveolar da região anterior da maxila e mandíbula com a medida FMA variando entre 20 e $30,1^{\circ}$, e a tabela 21 identifica que as espessuras ósseas póste-
Tabela 17 - Freqüência observada das medidas SA (subdivididas em três categorias), considerando a medida FMA (subdividida em duas categorias).

\begin{tabular}{|c|c|c|c|}
\hline \multirow{2}{*}{ SA } & \multicolumn{2}{|c|}{ FMA } & \multirow{2}{*}{ TOTAL } \\
\hline & $20.0^{\circ} / 25.0^{\circ}$ & $25.1^{\circ} / 30.1^{\circ}$ & \\
\hline $2,0 / 4,5 \mathrm{~mm}$ & $5(9,6 \%)$ & $10(19,3 \%)$ & $15(28,8 \%)$ \\
\hline $5,0 / 7,0 \mathrm{~mm}$ & $12(23,1 \%)$ & $15(28,8 \%)$ & $27(52,0 \%)$ \\
\hline 7,5 / 9,5 mm & $5(9,6 \%)$ & $5(9,6 \%)$ & $10(19,2 \%)$ \\
\hline TOTAL & $22(42,3 \%)$ & $30(57,7 \%)$ & $52(100 \%)$ \\
\hline
\end{tabular}

Tabela 19 - Freqüência observada das medidas IA (subdividida em três categoria), considerando a medida FMA (subdividida em duas categorias).

\begin{tabular}{|c|c|c|c|}
\hline \multirow{2}{*}{ IA } & \multicolumn{2}{|c|}{ FMA } & \multirow{2}{*}{ TOTAL } \\
\hline & $20.0^{\circ} / 25.0^{\circ}$ & $25.1^{\circ} / 30.1^{\circ}$ & \\
\hline $5,0 / 9,5 \mathrm{~mm}$ & $1(1,9 \%)$ & $13(25,0 \%)$ & $14(26,9 \%)$ \\
\hline $5,5 / 7,5 \mathrm{~mm}$ & $13(25,0 \%)$ & $14(26,9 \%)$ & $27(51,9 \%)$ \\
\hline $8,0 / 10,0 \mathrm{~mm}$ & $8(15,4 \%)$ & $3(5,8 \%)$ & $11(21,2 \%)$ \\
\hline TOTAL & $22(42,3 \%)$ & $30(57,7 \%)$ & $52(100 \%)$ \\
\hline
\end{tabular}

Tabela 21 - Teste de independência (qui-quadrado) entre as medidas SA, SP, IA, IP e a medida FMA.

\begin{tabular}{ccc}
\hline Teste & p-value & Dep (D) / Indep (I) \\
\hline SA x FMA & 0,674 & $\mathrm{I}$ \\
SP x FMA & 0,002 & $\mathrm{D}$ \\
IA x FMA & 0,003 & $\mathrm{D}$ \\
IP x FMA & 0,871 & $\mathrm{I}$ \\
\hline
\end{tabular}

ro-superior (SP) e ântero-inferior (IA) apresentaram relação de dependência com a medida FMA. A freqüência de pacientes com tendência ao crescimento vertical e redução de osso lingual ao ápice do incisivo central superior é maior quando comparada à freqüência de pacientes com tendência ao crescimento horizontal. A freqüência de pacientes com tendência ao crescimento vertical e redução de osso vestibular ao ápice do incisivo central inferior é maior quando comparada à freqüência de pacientes com tendência ao crescimento horizontal. 


\section{DISCUSSÃO}

O nível de quantidade óssea na região vestibular e lingual dos dentes anteriores superiores e inferiores são fatores limítrofes para o tratamento ortodôntico. Para detectar os níveis ósseos, vários métodos podem ser utilizados tais como tomografias computadorizadas, radiografias periapicais e telerradiografias laterais. Segundo Masumoto et al. ${ }^{14}$, a tomografia computadorizada é excelente método para detectar com precisão a quantidade de inserção óssea na região dos incisivos inferiores e superiores. Diversos trabalhos ${ }^{1,5,21}$ utilizaram cefalogramas laterais para avaliar a quantidade de osso na região dos incisivos. Segundo Hoeve e Mulie ${ }^{6}$, os ortodontistas não podem somente enfatizar a análise cefalométrica para determinar anatomicamente as limitações dos movimentos dentários. É importante a análise cuidadosa dos modelos de estudo e das radiografias periapicais da região a ser avaliada.

Com a modernização destes métodos, tornouse mais fácil para o ortodontista observar possiveis defeitos ósseos. Larato ${ }^{11}$ estudou deiscência e fenestração, e determinou que os dentes anteriores apresentam maior porcentagem de fenestrações e deiscências quando comparados aos dentes posteriores, sendo que, na mandíbula, os caninos foram os dentes mais associados a estes defeitos ósseos. Já Mulie e Hoeve ${ }^{16}$ relatam que qualquer dente pode apresentar fenestração ou deiscência, desde que sujeitos a forças pesadas e movimentos bruscos. Artun e Krogstad ${ }^{1}$ declaram que estudos experimentais, em macacos e cachorros, demonstraram que deiscência óssea pode ser produzida em osso alveolar quando os incisivos inferiores são movimentados anteriormente. Porém este resultado não foi corroborado por Wingard e Bowers ${ }^{22}$, que não encontraram fenestrações e deiscências nos dentes experimentais de humanos submetidos à inclinação vestibular.

O deslocamento apical total é um dos fatores, segundo Krol, Moll e Lundgren ${ }^{10}$, responsáveis pela reabsorção radicular. Lupi, Handelman e
Sadowsky ${ }^{12}$ afirmam que, entre diversos fatores, a densidade do osso alveolar está relacionada com a reabsorção radicular.

Para Martins et al. ${ }^{13}$, a movimentação dentária no sentido vestíbulo-lingual pode ocasionar perda da crista óssea alveolar e da inserção gengival, dependendo da espessura do osso alveolar e de fatores mecânicos, intensidade e velocidade da força aplicada e o tipo de movimentação dentária. Roberts ${ }^{17}$ declara que a iniciação e sustentação da resposta de reabsorção óssea determinam a velocidade do movimento dentário. Se todo osso oferecesse a mesma resistência ao movimento dentário, a ancoragem potencial dos molares superiores e inferiores seria a mesma. Entretanto, experiências clínicas mostram que os molares superiores geralmente têm valor menor de ancoragem que os molares inferiores no mesmo paciente. Isto ocorre porque as corticais finas e o osso trabecular da maxila oferecem menor resistência à reabsorção que as corticais espessas e trabéculas mais grossas do osso mandibular.

Para determinar a quantidade de inserção óssea, vários fatores devem ser considerados, como: gênero, idade do paciente, método radiográfico para avaliação do osso e tipo de má oclusão. A maioria dos autores ${ }^{5,11,12,18}$ utilizou em seus estudos pacientes adultos (com idade superior a 19 anos), portanto sem crescimento ósseo. Todavia, o trabalho conduzido por Ursi e McNamara Junior ${ }^{20}$ avaliou amostra de pacientes em idade de crescimento ósseo. O presente estudo foi realizado em pacientes com idade entre sete e 13 anos. Os resultados deste trabalho não evidenciaram relação de dependência entre a idade cronológica e a espessura do processo alveolar.

Steiner, Pearson, Ainamo ${ }^{19}$; Artun, Krogstad ${ }^{1}$; Wingard e Bowers ${ }^{22}$ concordam que os maiores fatores causadores da recessão gengival são o mal posicionamento dentário e a inclinação do dente no osso basal. Segundo Steiner, Pearson e Aina$\mathrm{mo}^{19}$, os dentes inferiores são mais acometidos quando comparados aos dentes superiores. 
Lupi, Handelman e Sadowsky ${ }^{12}$ declararam que a perda óssea ocorre em menor freqüência em pacientes adolescentes jovens, isto porque o tratamento é realizado às custas de crescimento da mandíbula e desenvolvimento dos processos alveolares. Para Ursi e McNamara Junior ${ }^{20}$ os crescimentos craniofaciais de pacientes com má oclusão de Classe II e I são muito semelhantes, sendo que as diferenças encontradas devem ser atribuídas ao crescimento na primeira década de vida. Segundo Bjornaas, Rygh e Boe ${ }^{2}$, pacientes portadores de severas más oclusões são mais susceptíveis à perda de suporte periodontal.

O trabalho de Handelman ${ }^{5}$ relata que a média da espessura do osso vestibular ao ápice do incisivo central superior é de $4,8 \mathrm{~mm}$ tanto para pacientes portadores de má oclusão Classe II como de Classe III esquelética.

O resultado do presente estudo evidenciou que a freqüência de pacientes Classe III $(36,8 \%)$ com redução de osso vestibular na maxila foi maior que nos pacientes Classe II $(24,2 \%)$.

Considerando que os pacientes portadores de má oclusão de Classe III esquelética tratados ortodonticamente requerem compensações dentárias, com inclinação dos incisivos superiores em direção vestibular, a espessura fina de osso nesta região não suportaria tal movimentação, tornando o tratamento ortodôntico inviável, sendo possível, apenas, se associado à cirurgia ortognática.

Outro aspecto definido por Handelman ${ }^{5}$ relaciona a altura facial, má oclusão e espessura da cortical óssea. Segundo o autor, os pacientes com má oclusão de Classe II e altura facial ântero-inferior aumentada, geralmente apresentam osso na lingual dos incisivos superiores de pequena espessura. Sendo, portanto insuficiente para corrigir sobressaliência excessiva. A espessura do osso na lingual do incisivo superior era maior em indivíduos de face curta, comparada a faces média e alta. $\mathrm{O}$ osso na cortical lingual dos ápices dos incisivos inferiores era maior no grupo de pacientes com face curta do que nos grupos com faces média ou alta. O osso vestibular ao ápice do incisivo inferior estava mais estreito no grupo de face longa do que nos grupos faces média e baixa. Massumoto et al. ${ }^{14}$ realizaram estudo semelhante, porém avaliaram a inclinação dos molares inferiores e a espessura da cortical mandibular. A inclinação do segundo molar inferior em indivíduos de face longa era significativamente menor que em indivíduos de faces curta e média. A espessura das corticais ósseas dos primeiros e segundos molares inferiores era maior em indivíduos de face curta quando comparadaaos indivíduos de faces longa e média.

O presente estudo demonstrou que pacientes com tendência de crescimento vertical (23\%), apresentam redução da espessura posterior do processo alveolar da região anterior da maxila (SP), quando comparados a pacientes com tendência ao crescimento horizontal $(5,8 \%)$. Portanto, pacientes com severa má oclusão de Classe II e tendência de crescimento vertical, que necessitam de retração ântero-superior, requerem atenção especial, pois a espessura reduzida do osso no lado palatino poderá implicar em fenestração ou reabsorção radicular.

Resultado semelhante foi observado nos pacientes com tendência de crescimento vertical $(15,4 \%)$, que apresentaram redução da espessura anterior do processo alveolar da região anterior $\mathrm{da}$ mandíbula (IA), quando comparados aos pacientes com tendência ao crescimento horizontal $(9,7 \%)$. Desta forma é imprescindível a avaliação da espessura óssea de pacientes com má oclusão de Classe II e tendência de crescimento vertical, sujeitos ao tratamento com compensação dentária, ou seja, vestibularizando os dentes ântero-inferiores, para evitar fenestração ou perda óssea.

Cuidados semelhantes requerem os pacientes Classe III submetidos à cirurgia ortognática, os quais geralmente necessitam de preparo ortodôntico pré-cirúrgico visando a descompensação dentária, ou seja, inclinando para vestibular os incisivos lingualizados. 


\section{CONCLUSÕES}

Fundamentado nas evidências encontradas, considerando as condições em que este estudo foi realizado, algumas conclusões podem ser formuladas.

1) A relação entre a espessura posterior do processo alveolar da região anterior da maxila (SP) com as Classes II e III esquelética é de independência. Todavia a medida SA (osso vestibular ao ápice do incisivo central superior) relacionada

às Classes II e III esqueléticas apresentou relação de dependência, sendo que $36,8 \%$ dos pacientes Classe III apresentam a espessura SA diminuída medindo entre 2,5 a 4,5 mm.

2) Não foi observada relação de dependência entre a espessura do processo alveolar da região anterior da mandíbula (IA, IP) com o tipo de má oclusão esquelética (Classe II e III esquelética).
3) Foi observada relação de independência entre a espessura do processo alveolar da região anterior da maxila e mandíbula com as idades dos pacientes.

4) Foi observada relação de dependência entre a espessura posterior do processo alveolar da região anterior da maxila (SP) e a espessura anterior do processo alveolar da região anterior da mandíbula (IA) com a medida FMA.

5) Foi observada relação de dependência entre a espessura posterior do processo alveolar da região anterior da maxila (SP) e a espessura anterior do processo alveolar da região anterior da mandíbula (IA) com a medida NSGn.

Enviado em: Junho de 2004 Revisado e aceito: Abril de 2005

Thickness of the alveolar process in the anterior region of the maxilla and mandible of patients with antero-posterior discrepancy

\begin{abstract}
The purpose of the present study was to assess the thickness of the alveolar process on the anterior region of maxilla and mandible of patients with antero-posterior discrepancy. The thickness of the alveolar process on the anterior regions of maxilla and mandible was measured by lateral cephalograms from fifty two patients with ages between seven to thirteen years. All the patients included on this study had the mandibular plan angle between 20 and 30 degrees and antero-posterior bone discrepancy between maxilla and mandible. The statistical analysis (Chisquare test) revealed independence between the thickness of the maxilla and mandible alveolar process and the patient age. However, a statistical dependence was found between the type of the malocclusion and the thickness of the buccal bone on anterior region of maxilla. The patients with Class III malocclusion showed a higher percentage of buccal bone reduction on the anterior region of maxilla when compaired with Classe II patients. The patients with trend to vertical growing had a reduced dimension of the maxilla lingual bone and mandible buccal region.
\end{abstract}

Key words: Thickness of the alveolar process. Malocclusion. Face type. latrogenic loss. 


\section{REFERÊNCIAS}

1. ARTUN, J.; KROGSTAD, O. Periodontal study of mandibular incisors following excessive proclination. Am J Orthod Dentofacial Orthop, St. Louis, v. 91, no. 8, p. 225-232, 1987

2. BJORNAAS, T.; RYGH, P.; BOE, O. E. Severe overjet and overbite reduced alveolar bone height in 19-year-old men. Am J Orthod Dentofacial Orthop, St. Louis, v. 106, no. 3, p. 139-145, 1994.

3. EDWARDS, J. G. A study of the anterior portion of the palate as it relates to orthodontic therapy. Am J Orthod, St. Louis, v. 69, no. 3, p. 249-73, Mar. 1976.

4. HALAZONETIS, D. J. Experimentos com computador utilizando um modelo bidimensional das estruturas de suporte dentário. R Dental Press Ortodon Ortop Maxilar, Maringá, v. 2, n. 2 , p. 68-69, mar./abr. 1997.

5. HANDELMAN, C. S. Processo alveolar da região anterior: importância na limitação do tratamento ortodôntico e influência na ocorrência de seqüela iatrogênica. R Dental Press Ortodon Ortop Maxilar, Maringá, v. 1, n. 1, p. 38-51, set./ out. 1996.

6. HOEVE, A. T.; MULIE, R. M. The effect of antero-postero incisor repositioning on the Palatal Cortex as studied with laminagraphy. J Clin Orthod, Boulder, v. 10, no. 11, p. 804-822, Nov. 1976.

7. INTERLANDI, S. Análises cefalométricas I: cefalogramas. In: Ortodontia: bases para a iniciação. 4. ed. São Paulo, 1999. cap. 28. p. 667-710

8. ISHIKAWA, H.; NAKAMURA, S.; IWASAKI, H.; KITAZAWA, S.; TSUKADA, H.; CHU, S. Dentoalveolar compensation in negative overjet cases. Angle Orthod, Appleton, v. 70, no. 2, p. 145-148, 2000.

9. ISHIKAWA, H.; NAKAMURA, S.; IWASAKI, H.; KITAZAWA S.; TSUKADA, H.; SATO, Y. Dentoalveolar compensation related to variations in sagittal jaw relationships. Angle Orthod, Appleton, v. 69 , no. 6, p. 534-538, 1999.

10. KUROL, J.; MOLL, O.; LUNDGREN, D. Reabsorção radicular apical em adultos tratados ortodonticamente. R Dental Press Ortodon Ortop Maxilar, Maringá, v. 2, n. 2, p. 46-47, mar./abr. 1997.
11. LARATO, D. C. Alveolar plate fenestrations and dehiscences of the human skull. Oral Surg, v. 29, no. 6, p. 816-819, June 1970.

12. LUPI, J. E.; HANDELMAN, C. S.; SADOWSKY, C. Prevalence and severity of apical root resorption and alveolar bone loss in orthodontically treated adults. Am J Orthod Dentofacial Orthop St. Louis, v. 109, no. 1, p. 28-37, 1996.

13. MARTINS, P. P; GARIB, D. G.; GREGHI, S. L. G; HENRIQUES, J. F. C. Avaliação periodontal dos incisivos inferiores em pacientes tratados ortodonticamente com extrações quatro de pré-molares. Rev Fac Odontol Bauru, Bauru, v. 10, n. 4, out./dez. 2002.

14. MASUMOTO, T.; HAYASHI, I.; KAWAMURA, A.; TANAKA, K.; KASAI, K. Relationships among facial type, buccolingual molar inclination, and cortical bone thickness of the mandible. Eur $\mathbf{J}$ Orthod, London, v. 23, p. 15-23, 2001.

15. MEYER, R.; KRUEGER, D. A minitab guide to statistics. 2nd ed. [S.I. : s.n.], 2001.

16. MULIE, R. M.; HOEVE, A. T. The limitations of tooth movement within the symphysis. J Clin Orthod, Boulder, v. 10, no. 12, p. 882-893, Dec. 1976.

17. ROBERTS, W. E. Biomecânica, metabolismo e fisiologia óssea na prática ortodôntica. In: GRABER,T. M.; VANARSDALL, R. L. Ortodontia: princípios e técnicas atuais. 2. ed. Rio de Janeiro: Guanabara Koogan, 1994. cap. 3, p. 175-212.

18. SPERRY, T. P.; SPEIDEL, T. M.; ISAACSON, R. J.; WORMS, F. W. $W$. The role of dental compensations in the orthodontic treatment of mandibular prognathism. Angle Orthod, Appleton, v. 47, no. 4, p. 293-295, Oct. 1977.

19. STEINER, G. G.; PEARSON, J. K.; AINAMO, J. Changes of the marginal periodontium as a result of labial tooth movement in monkeys. J Periodontol, Chicago, v. 52, p. 314-320, June 1981.

20. URSI, W.; MCNAMARA Junior, J. Crescimento craniofacial em pacientes apresentando maloclusões de Classe II e maloclusões normal, entre os e os 10 e os 12 anos de Idade. Rev Dental Press Ortodon Ortop Maxilar, Maringá, v. 2, n. 5, p. 49-59, set./out. 1997

21. WEHRBEIN, H.; BAUER, W.; DIEDRICH, P. Incisivos inferiores, osso alveolar e sínfise após tratamento ortodôntico: um estudo retrospectivo. R Dental Press Ortodon Ortop Maxilar Maringá, v. 2, n. 4, p. 21-27, jul./ago. 1997.

22. WINGARD, C. E.; BOWERS, G. M. The effect on bone from facial tipping of incisors in monkeys. J Periodontol, Chicago, v. 47 p. 450-455, Aug. 1796.
Endereço para correspondência

Rachel de Mattos Garcia

Avenida Emílio Winther, 1450, apto. 13, Independência

CEP: 12.030-001 - Taubaté/SP

E-mail: garcia.rachel@ig.com.br 